\title{
Special Issue on Computer-Assisted Craniofacial Reconstruction and Modeling
}

\author{
Guest Editor: Thorsten M. Buzug \\ Department of Mathematics and Technology, RheinAhrCampus Remagen, Germany
}

\section{Editorial}

The reconstruction of facial soft tissue is an essential processing phase in the fields of forensics, anthropology as well as maxillofacial surgery. In the first two areas the working subject is a skull find of a deceased where the soft tissue needs to be reconstructed. In forensics, this work helps to identify skeletons from open cases of death. In anthropology, the comparison of facial features between modern and ancient human beings is the main focus of research. Finally, in the case of maxillofacial surgery, a face must be reconstructed after, for example, cancer surgery. The goals of forensics, anthropology and maxillofacial surgery differ substantially. However, the mathematical basis of computer-aided soft facial reconstruction is effectively the same.

Keywords: forensics, anthropology, maxillofacial surgery, 3D virtual interaction, face reconstruction, soft tissue modeling

\section{Forensic Face Reconstruction}

"Mystery of dead woman in Winkeler Bay solved" - After ten years, a female body found mutilated beyond recognition has been identified using a technique known as facial reconstruction. Thanks to the information obtained as a result of the reconstruction, the search for the potential murderer has now been initiated. Reports of this type were rare in the past. Today, however, a revolutionary progress in computeraided methods has also made its way into the reconstruction of the soft tissues of the human face.

The main goal of computer-based craniofacial reconstructions is to simplify and speed up the process of finding the appropriate reproduction of a person's facial appearance corresponding to forensic findings. The recent developments in the IT sector and the resulting improvement of computing power and progress in technologies for visualization, lead to novel ways for computer-assisted forensic face reconstruction.

Based on the findings of traditional facial reconstruction, more and more complex software programs are being designed and applied. In combination with state-of-the-art medical imaging and laser scanning technology, detailed 3Dimages can be created with different facial expressions.

In general, according to R.P. Helmer [1] the classic manual reconstruction method follows four basic steps.

\section{i) Examination of the skull}

The examination of the skull includes the determination of key skull dimensions, the registration and recording of the characteristic shapes of the skull bones (see Fig. 1a), the preparation of orthogonal drawings of the skull's norma frontalis and lateralis, preparation of sketches of pre-planned soft tissue profiles, as well as the photographic documentation of the skull.

\section{ii) Development of a Reconstruction Plan}

Within this step, all data obtained during the investigation are compiled and organized. These data include all results of the forensic, anthropological and dentological analysis of the corpse's remains, as well as findings obtained by the criminal investigation department at the site where the corpse was found- 


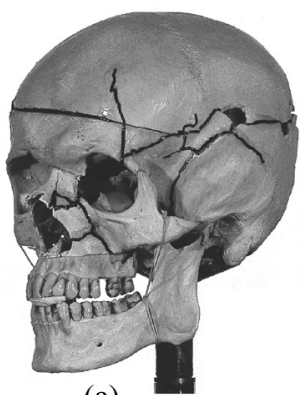

(a)

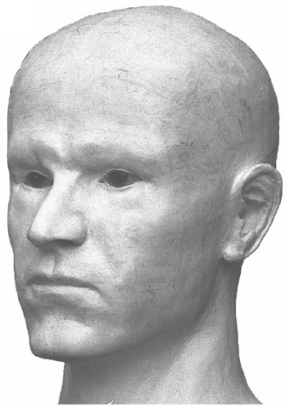

(c)

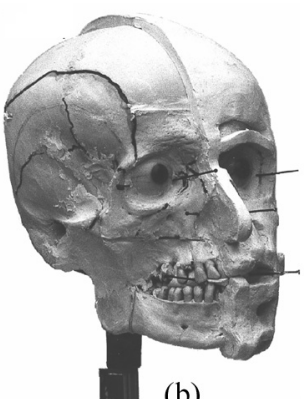

(b)

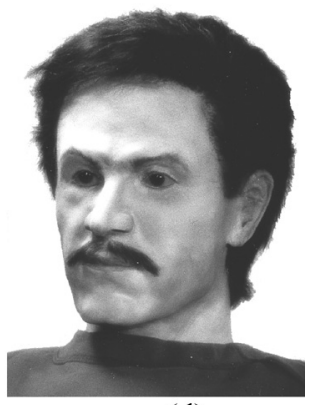

(d)
Fig. 1. Illustration of the four main processing steps of the classic manual reconstruction technique (courtesy of R.P. Helmer [1]).

especially samples of hair and dental prostheses. Additionally, soft tissue depths for specified points on the face and head are listed in accordance with the individual's gender and ables and established in terms of magnitude according to the type of constitution involved.

\section{iii) Practical Sculpturing}

The shapes of the large masticatory muscles are modeled using wax on both sides with the skull and lower jaw mounted on a tripod. After that, shell-shaped glass eyes are embedded in wax in the eye sockets (see Fig. 1b). This is followed by an application of modeling wax combs to the thickness specified for the respective facial soft tissue, initially just on the left-hand side of the skull. After that, the nose, mouth and eyelids are modeled. Once the left-hand side of the face is complete, the right-hand side is modeled and the entire face reworked finely to achieve a natural appearance. Ears are modeled as add-ons at the appropriate positions (see Fig. 1c). After that, the head is mounted on a base board by means of a short fixing rod and reconstruction is completed by modeling the neck and shoulder-tops as add-ons.

\section{iv) Mask Design}

Make-up can be applied to the reconstructed wax faces to achieve a lifelike and natural appearance. An experienced make-up artist should be engaged to produce a natural-looking hairstyle tailored to the head's dimensions, attach eyebrows and, if necessary, a beard, and lend the face a natural-looking skin color including red lips (see Fig. 1d). During layout design for facial reconstruction, it is important to note that the fundamental objective is not necessarily the realization of a "handsome" face, but a reproduction of the unknown person's individual facial features to the highest possible accuracy.

These four key steps may be translated into the following computer-assisted reconstruction steps.

\section{i’) Computed Tomography Scan of the Skull}

For digitalization of a skull find computed tomography (CT) may be used as one modality (see Fig. 2a-c). Beyond medical application, CT imaging has become established in forensic and anthropologic sciences as well since it provides excellent support for the methods of facial reconstruction. Especially, the digital three-dimensional CT image (see Fig. 2c) offers the advantage that even very complex geometric features of the skull form, e.g. so-called "crest lines" or other structural characteristics defined by differential geometry, may be determined.

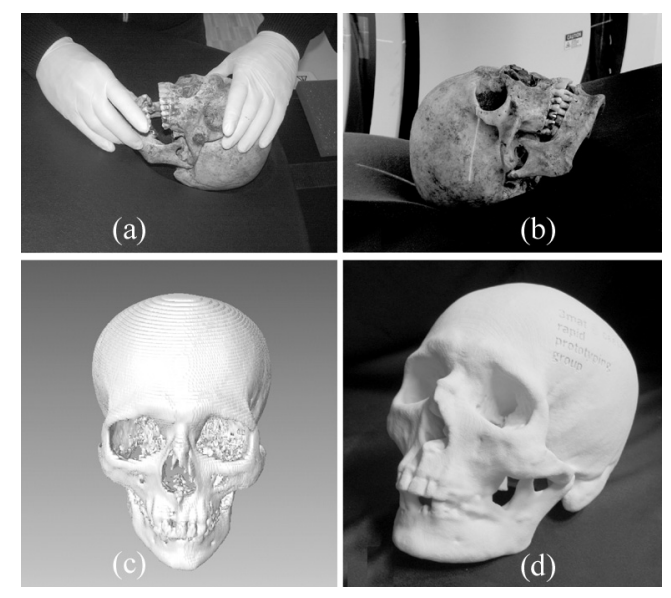

Fig. 2. From CT imaging and virtual 3D visualization to the rapid prototyping model. Picture (d) of rapid prototyping cast: Courtesy of H. Seitz and C. Tille [2]. 


\section{ii’) Matching of a Soft Tissue Template}

The development of a reconstruction plan aims at identifying an appropriate soft tissue template from a database using additional retrieving information (see Fig. 3a). This information - which should originate from the results of the forensic and anthropological examination (e.g. hair fragments) at the place where the body was discovered - is still indispensable and is also used in the preparation of step iv'. A matching of the anatomical landmarks of a soft tissue template with anatomical landmarks of the skull find may be carried out. This process can be supported by virtual soft tissue dowels (see Fig. 3b) that can be placed onto the iso-gray value surface of the CT data. A soft tissue template database may consist of MRI, low-dose CT or facial laser scans.

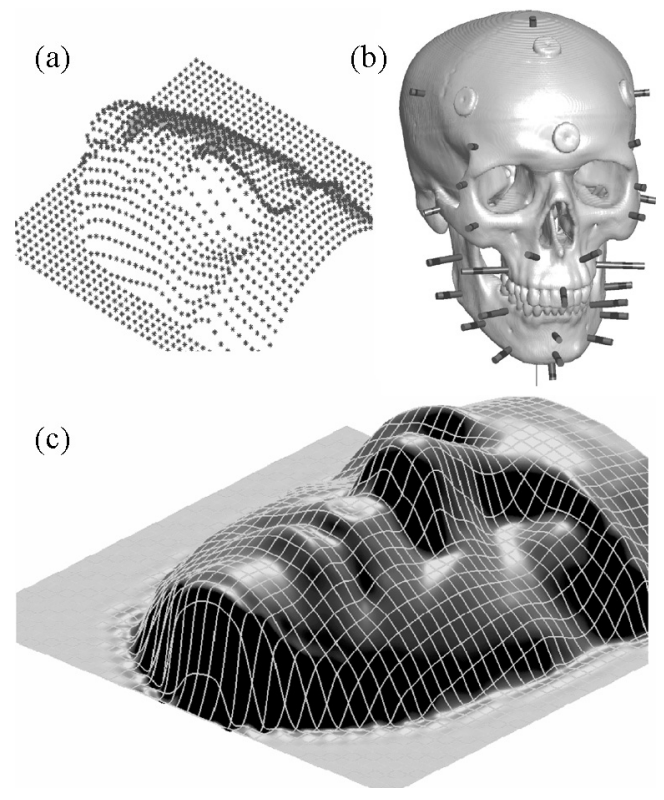

Fig. 3. Example of a computer-assisted facial reconstruction.

\section{iii') Warping of Template onto Skull Find}

Within this step a (potentially elastic) registration of the template with the skull is carried out based on anatomical landmarks and, if necessary, crest lines or other structural characteristics and statistics. Usually, subsequent interactive corrections of individual parts of the face are necessary (see Fig. 3c).

\section{iv') Texture Mapping: Virtual Make-Up}

Texture mapping includes application of patterns, shading and colors to surfaces. Today, computers are very well able to carry out this task. However, the problem is rather the scope for artistic design. As in the manual method, mentioned under step iv, the decisions in this field are still left to the medico-legal expert and the anthropologist. But decision making can be supported by the computer, and wrong decisions can easily be corrected.

The classic manual procedure (steps $i$-iv) is not called into question by the new techniques (steps $i$ '- $\left.i v^{\prime}\right)$. On the contrary, these techniques aim at supplementing and, above all, at accelerating traditional procedures.

\section{Anthropologic Face Reconstruction}

An archaeological or anthropological example of facial reconstruction, respectively, is given in Fig. 4. It is the facial reconstruction of the popular Egyptian mummy of the Centre for Biological Egyptology at Manchester University. The face of the Egyptian, who lived during the 19th dynasty more than 3000 years ago, was reconstructed using modern computer-assisted modeling techniques. As mentioned in the section above, the first step of the processing chain was the acquisition of CT data of the specimen (see Fig. 4a).

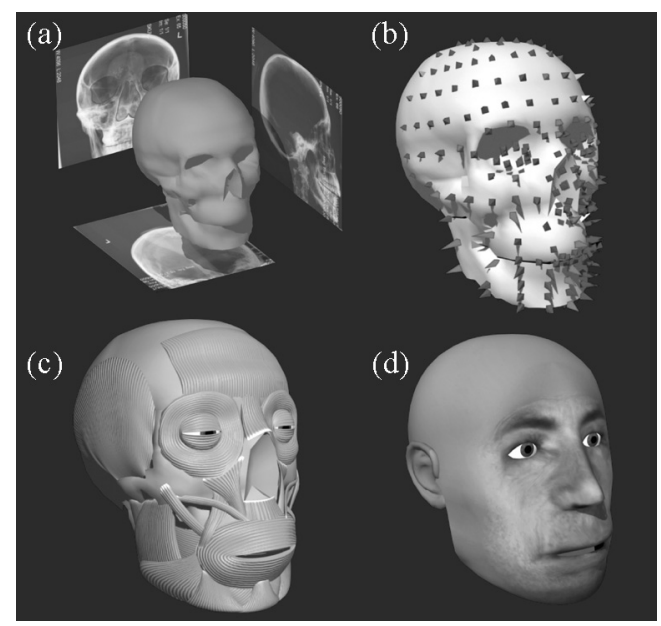

Fig. 4. Archaeological face reconstruction (Bolton Mummy: Courtesy of M. Evison and D. Schofield [4]). 
The virtual procedure shown here differs from steps $i i$ ' and iii'. In the second step, soft tissue depth landmarks were placed at characteristic coordinates (see Fig. 4b). Then, muscles were modulated (Fig. 4c) and in a final step the skin was rendered (Fig. 4d). This chain is an exact translation of the manual to a virtual procedure. The anatomical face reconstruction was carried out by $D$. Schoffield and M. Evison [3] from Nottingham and Sheffield University, respectively.

Generally, there are two procedural alternatives to working with the $\mathrm{CT}$ data. As one alternative, which has been described in points $i^{\prime}$ ) to $\left.i v^{\prime}\right)$ and the examples above, it is of course conceivable to use the three-dimensional CT data as the basis of a virtual soft tissue reconstruction, especially since it is stored in the computer as a representation that can be directly used for further work. The other alternative uses the CT data as the basis for the so-called rapid prototyping technique that produces plastic copies of the skull in its true size (see Fig. 2d); to these the anthropologist can add the soft tissues with traditional plasticine without putting the archaeological find at risk.

Anthropologists are interested in giving an impression of the appearance of famous persons in history or an idea of a Neanderthaler's visage as an artwork for a museum's exhibition. Forensic anthropologists, however, are working hand in hand with criminal departments to identify a person by means of a soft-facial reconstruction to be published. While the first goal offers some artistic freedom to the sculptor, the latter restricts him or her to information that has been assembled within the criminal investigation.

\section{Craniofacial Surgery}

There is a difference between the objectives of craniofacial or facial plastic surgery on the one hand and forensic or anthropologic face reconstruction on the other. In the case of the latter two application, fields the reconstruction of facial soft tissue must be performed without any knowledge of the exact target face. The main goal is to identify an unknown individual (in the forensic case) or at least to give an impression of the visual appearance of historic persons (in the anthropologic case). As mentioned above, the latter type of problems leaves some degree of artistic freedom to the sculptor.

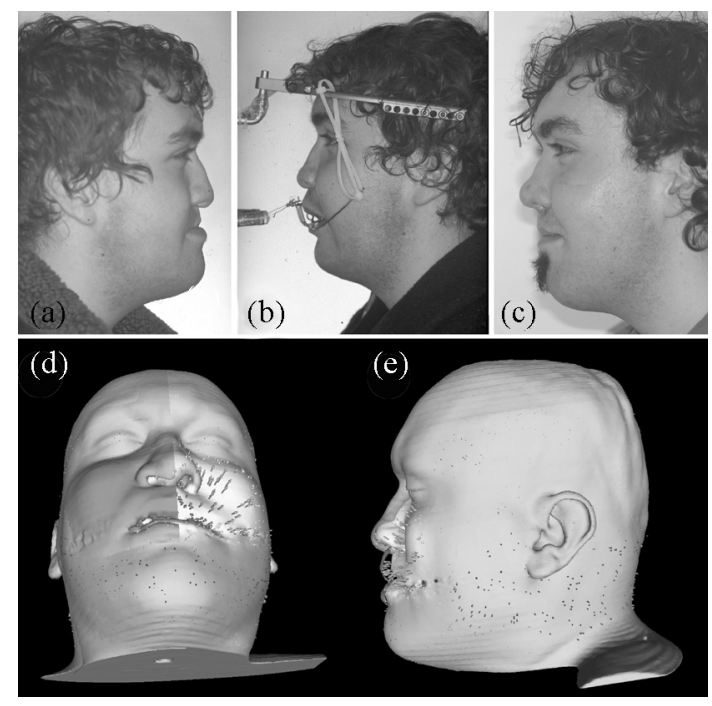

Fig. 5. Illustration of craniofacial intervention: Lengthening the maxilla by distraction osteogenesis (courtesy of T. Hierl [3]).

Conversely, in the case of craniofacial or facial plastic surgery, the main goal is to reestablish a harmonic facial appearance of a patient, e.g. after tumor resection. Another medical application scenario is maxillary distraction osteogenesis (DOG). Soft tissue must be simulated to predict the result of the procedure. However, since the goals are different, the methods of soft tissue simulation on the basis of underlying bones are quite similar.

Figure $4 a / b$ shows a sample DOG, a procedure in the treatment of severe maxillary hypoplasia and retrusion [3]. Up to now, most investigations have focused on questions regarding the procedure, bony changes, and dental occlusion. However, it is also important to study the concomitant soft tissue changes associated with large bony maxillary advancements as well.

As an example of DOG, the group of T. Hierl et al. [3] compared pre- and post-treatment CT scans using a tool chain based on rigid and nonrigid registrations which have been evaluated for bony changes in time-series analysis. This chain allows the extraction of data for individual anatomical landmarks and displays changes in a color pattern (see Fig. 4 d/e). Furthermore, soft tissue and bony changes could be compared. Facial appearance was altered towards a harmonic relation (see Fig. 4c). 


\section{Structure of the Issue}

The special issue is structured as follows. The first three papers describe directions of computerassisted soft-facial reconstruction in forensic sciences. The next two papers show how anthropological, as well as surgical face reconstruction benefit from virtual procedures. The issue is completed by two case-study papers on surgical face reconstruction.

In the first paper, A Multi-Modality ComputerAided Framework Towards Postmortem Identification by A. Mang et al., a framework based on computed tomography (to acquire a virtual representation of the skull find) and on magnetic resonance imaging (providing the desired soft tissue information to produce facial soft tissue templates) is presented. Additionally, a prototype of a forensic workplace is described.

The second paper, Statistically Deformable Face Models for Cranio-Facial Reconstruction by P. Claes et al., stresses the fact that template-based reconstruction is biased by the specific choice of the template. The authors reduce this bias by using a flexible statistical model of a dense set of facial surface points combined with an associated sparse set of skull landmarks.

In the paper 3D Semi-Landmarks Based Statistical Face Reconstruction by M. Berar et al., it is proposed first to build a 3D statistical model of the set skull/face from 3D CT scans. The authors introduce a reconstruction method in order to estimate, from this statistical model, the 3D facial shape of one person from known skull data.

The next paper focuses on the anthropological application of modern face reconstruction techniques. The paper Using Image Analysis Software to Create a Physical Skull Model for the Facial Reconstruction of a Wrapped Akhmimic Mummy by H. Gill-Robinson et al. shows the reconstruction of mummy faces on the basis of computed tomography scans. The authors provide an analysis and interpretation of twodimensional CT images through the preparation of virtual on-screen and physical threedimensional models.

The paper Computer-Assisted Planning in CranioMaxillofacial Surgery by S. Zachow et al. presents a computer-assisted face modeling, planning and simulation approach that allows for preoperative assessment of different therapeutic strategies on basis of three-dimensional patient models. The resulting facial appearance is simulated via finite-element methods on the basis of a biomechanical tissue model, and visualized using high-quality rendering techniques.

The next two papers give examples of a surgeon's view on facial reconstructions. In the first paper, CAD-CAM Implants in Esthetic and Reconstructive Craniofacial Surgery by T. Hierl et al., the authors show how CAD-CAM techniques are introduced to bilaterally affected skulls in esthetic reconstructive surgery. They demonstrate that surgery could become less invasive and results more predictable. A tool chain is shown to generate implants on a scientific basis.

The last paper in this special issue, Computer Assisted Oral and Maxillofacial Reconstruction by A. Schramm et al., evaluates the benefit and the indications of computer-assisted surgery in the treatment of cranio-maxillofacial surgery. Preoperatively required soft and hard tissues were measured using the mirrored data set of the unaffected side; size and location of the graft were chosen virtually. Contours of transplanted tissues were intra-operatively navigated to the preoperatively simulated reconstructive result. The authors show that interventional navigation makes tumor and reconstructive surgery more reliable.

\section{Acknowledgments}

I would like to thank Richard Helmer (Institute of Applied Forensic Medicine Remagen), Herman Seitz, Carsten Tille (caesar Bonn), Damian Schofield (University of Nottingham), Martin Evison (University of Sheffield) and Thomas Hierl (University of Leipzig) for supporting this editorial with illustrations. I would like to thank my colleagues Georg Schmitz (University of Bochum), Karin Gruber (University of Koblenz), Peter Claes (University of Leuven), Maxim Berar (Laboratoire des Images et des Signaux, Saint Martin d'Hères), Stefan Zachow (Zuse Institute Berlin) as well as Jens Bongartz, Ulrich Hartmann and Dietrich Holz 
(RheinAhrCampus Remagen) for reviewing the papers of this issue. In addition to thanking all the contributors for their cooperation, I would like to acknowledge the valuable help of the Editor-in-Chief, Sven Loncaric, who continuously supported this special issue of CIT.

\section{References}

[1] R.P. Helmer, T.M. Buzug ANd P. HeRING, Plastic Facial Reconstruction on the Skull — A Transition in Germany from a Conventional Technique to a new one, Proceedings of the First International Conference on Reconstruction of Soft Facial Parts, K.M. Sigl et al. (Eds.), (Pulished by Bundeskriminalamt KI 21-Z, Wiesbaden, 2003) pp. 75-90.

[2] K. Raic, T. JANSEN, B. VON RYMON-LiPINSKI, C. Tille, H. Seitz And E. Keeve, Fast generation of stereolithographic models, Biomed Tech 47, Suppl 1, Pt 1 (2002) pp. 83-85

[3] M. Evison, S. DAVEY, J. MARCH AND D. SCHOFIELD, Computational Forensic Facial Reconstruction, Proceedings of the First International Conference on Reconstruction of Soft Facial Parts, K.M. Sigl et al. (Eds.), (Pulished by Bundeskriminalamt KI 21-Z, Wiesbaden, 2003) pp. 29-34.

[4] T. Hierl, N. Klisch, R. KLÖPPEL AND A. HEMPRICH, Therapie ausgeprägter Mittelgesichtsrücklagen mit Hilfe der Distraktionsosteogenese, Mund Kiefer GesichtsChir 7 (2003) pp. 7-13.

Contact address: Thorsten M. Buzug Department of Mathematics and Technology RheinAhrCampus Remagen Südallee 2, D-53424 Remagen Germany e-mail: buzug@rheinahrcampus.de

Thorsten M. Buzug is a Professor in the Department of Mathematics and Technology, RheinAhrCampus Remagen, Germany. He received his Diplom-Physiker degree in 1989 and his Ph.D. in 1993 in applied physics, both from the Christian-Albrechts-University Kiel, Germany, where he worked in the field of signal processing applied to chaotic systems. From 1993 to 1994 he had a postdoctoral position at the German Federal Armed Forces Underwater Acoustics and Marine Geophysics Research Institute, where he worked on image acquisition and processing techniques for SONAR applications. At the end of 1994 he joined the Philips Research Laboratories Hamburg, Germany. He was the leader of the Philips Research Cluster Medical Image Processing and responsible for several projects in that field. In October 1998 he was appointed as professor of physics and medical engineering at the RheinAhrCampus Remagen, Germany. He is a member of the German Physical Society (DPG), the German Society of Biomedical Engineering (DGBMT), the German Society of Nondestructive Testing (DGfZP) and the IEEE. 